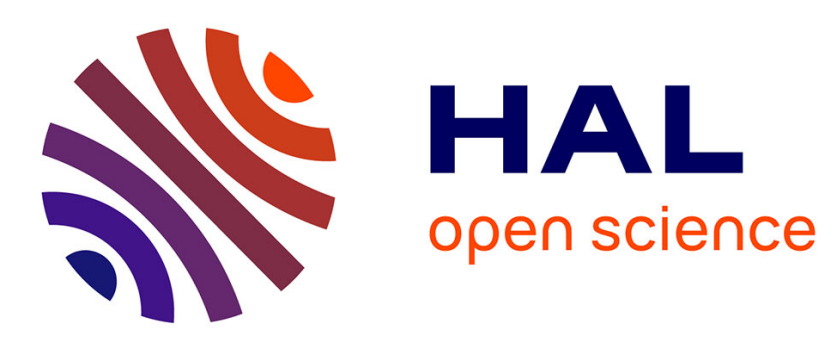

\title{
The role of pair dispersion in turbulent flow
}

Mickaël Bourgoin, Nicholas T. Ouellette, Haitao Xu, Jacob Berg, Eberhard

Bodenschatz

\section{To cite this version:}

Mickaël Bourgoin, Nicholas T. Ouellette, Haitao Xu, Jacob Berg, Eberhard Bodenschatz. The role of pair dispersion in turbulent flow. Science, 2006, 311 (5762), pp.835-838. 10.1126/science.1121726 . hal-00492363

\section{HAL Id: hal-00492363 https://hal.science/hal-00492363}

Submitted on 23 Jun 2010

HAL is a multi-disciplinary open access archive for the deposit and dissemination of scientific research documents, whether they are published or not. The documents may come from teaching and research institutions in France or abroad, or from public or private research centers.
L'archive ouverte pluridisciplinaire HAL, est destinée au dépôt et à la diffusion de documents scientifiques de niveau recherche, publiés ou non, émanant des établissements d'enseignement et de recherche français ou étrangers, des laboratoires publics ou privés. 


\title{
The Role of Pair Dispersion in Turbulent Flow
}

\author{
Mickaël Bourgoin, ${ }^{1}$ Nicholas T. Ouellette ${ }^{2}$ Haitao $\mathrm{Xu},{ }^{2,3}$ \\ Jacob Berg, ${ }^{4}$ Eberhard Bodenschatz ${ }^{2,3, *}$ \\ ${ }^{1}$ Laboratoire des Écoulements Géophysiques et Industriels-C.N.R.S. (U.M.R. 5519), \\ BP 53-38041, Grenoble Cedex 9, France \\ ${ }^{2}$ Laboratory of Atomic and Solid State Physics, Cornell University, \\ Ithaca, NY 14853, USA \\ ${ }^{3}$ Max Planck Institute for Dynamics and Self-Organization, \\ Göttingen, Germany \\ ${ }^{4}$ Ris $\emptyset$ National Laboratory, \\ DK-4000 Roskilde, Denmark
}

*To whom correspondence should be addressed; E-mail: eberhard.bodenschatz@ds.mpg.de.

Mixing and transport in turbulent flows, relevant in a huge variety of both natural and industrial systems including chemical reactors, combustion engines, warm clouds, and biological odor detection, depend strongly on local concentration fluctuations. Local concentration in turn is intimately tied to the longstanding problem of the average spreading rate of pairs of fluid elements. We have measured this separation rate in an intensely turbulent laboratory flow and have found excellent agreement with the seminal theoretical work of Batchelor, who predicted that the initial separation of the pair plays an important role in the subsequent spreading of the particles. When the initial separation is treated as a parameter, the power law scaling of the separation rate changes with surprising consequences for the decay of the concentration 


\section{fluctuations of substances carried along with the flow.}

Turbulent mixing of liquids and gasses is ubiquitous in nature (1): it is the basis of all industrial fluid mixing processes, and it determines the spread of pollutants or bioagents in the atmosphere (2) and oceans (3). Biological organisms in marine ecosystems exploit it for their survival (4-6). A crucial component of turbulent mixing is the fluctuation of local concentration. The rate of destruction of ozone in the atmosphere, for example, is largely determined by these fluctuations rather than by the mean concentration (7), as is the toxicity of gas leaks or air pollution. It is natural to relate these concentration fluctutations to the separation of two nearby fluid elements, i.e., pair dispersion $(8,9)$.

In a quiescent fluid, the relative dispersion of two fluid elements (or tracer particles) is dominated by diffusion. The particles undergo Brownian motion, and the mean-square separation between them grows linearly in time. In a turbulent flow, however, if the two particles are separated by distances smaller than the characteristic size of the largest eddies in the flow, they will separate faster (i.e., superdiffusively). At large separation times and distances, the local correlations responsible for the superdiffusive separation will no longer be present, and, on the average, the relative dispersion will again be linear in time.

Despite almost eighty years of scientific inquiry into relative dispersion (2,9-17), no clear experimental verification of the theoretical predictions has emerged. One critical unresolved question is the extent to which the initial separation of the fluid particles influences their subse-

quent motion. Surprisingly, our measurements in a laboratory water flow $(18,19)$ in very intense turbulence suggest that the initial separation remains important for all but the most violent flows on Earth. This observation has important consequences for such varied problems as pollution control, combustion modelling, hazardous chemical control, and even the understanding of how animals locate food, predators, and mates $(5,6)$.

We have measured relative dispersion in a water flow at high turbulence levels using op- 
tical particle tracking. This technique has been used for a number of years in turbulence research $(13,20)$, but was limited to the measurement of low turbulence level flows due to the fact that tracer particle motions must be resolved over times comparable to the smallest timescale of the turbulence (i.e., the Kolmogorov time scale $\tau_{\eta}=(\nu / \epsilon)^{1 / 2}$, where $\nu$ is the kinematic viscosity and $\epsilon$ is the energy dissipation rate per unit mass). In intense turbulence these times are often very small. The turbulence level is generally quantified by the Reynolds number, which measures the ratio of the nonlinear inertial forces to the linear viscous forces. In the present work, we report the Reynolds number based on the Taylor microscale, $R_{\lambda}=\sqrt{15 u^{\prime} L / \nu}$, where $u^{\prime}$ is the root-mean-square velocity of the turbulent fluctuations and $L$ is the largest length scale of the turbulence. In our water flow at $R_{\lambda}=815$, the highest Reynolds number reported in this work, $\tau_{\eta}=0.54 \mathrm{~ms}$, and so very fast detectors must be used to resolve the fine structure of the flow. Previously, using silicon strip detectors from high energy physics $(18,19)$ we extended the particle tracking technique to flows with high turbulence levels. Such detectors, however, are unsuitable for measuring the statistics of many tracer particles at once. Here we use three Phantom v7.1 digital cameras from Vision Research, Inc., which record 27,000 pictures per second at a resolution of $256 \times 256$ pixels, as sketched in Fig. 1a. We can use this camera system to track several hundred particles at once (21). An example of two such simultaneously measured particle tracks is shown in Fig. 1 b.

We generate turbulence between coaxial counter-rotating baffled disks in a closed chamber with a volume of approximately $0.1 \mathrm{~m}^{3}$, as shown in Fig. 1a. We make measurements in a subvolume of roughly $5 \times 5 \times 5 \mathrm{~cm}^{3}$ in the center of the tank where the mean flow is statistically zero. Polystyrene tracer particles $25 \mu \mathrm{m}$ in diameter, comparable to the Kolmogorov length scale $\eta=\left(\nu^{3} / \epsilon\right)^{1 / 4}$, the smallest scale of the turbulence, are illuminated by two frequencydoubled, pulsed Nd:YAG with a combined power of roughly $150 \mathrm{~W}$. The particle positions are measured with a precision of roughly 0.1 pixels (21), corresponding to about $20 \mu \mathrm{m}$ in the flow. 
Further description of this flow has been reported previously $(18,19)$.

By analyzing our measured particle tracks, we have investigated the time evolution of the mean-square separation between two fluid elements. Predictions for the superdiffusivity of this pair dispersion in turbulence date back to 1926 when Richardson (10) suggested that it should grow in time as $t^{3}$. By applying Kolmogorov's scaling theory (22), Obukhov (23) specified that, in the inertial range of turbulence where the only relevant flow parameter is the energy dissipation rate per unit mass $\epsilon$, the pair dispersion should grow as $g \epsilon t^{3}$, where $g$ is a universal constant. Batchelor (11) refined this work, predicting that the mean-square separation should grow as $t^{2}$ for times shorter than a characteristic timescale $t_{0}$ that depends on the initial separation of the pair.

Defining $\Delta(t)$ as the separation of two fluid elements at time $t$ and $\Delta_{0}$ as the initial separation between the fluid elements, Batchelor predicted that, for $\Delta_{0}$ in the inertial range,

$$
\left\langle\left[\vec{\Delta}(t)-\vec{\Delta}_{0}\right]^{2}\right\rangle=\frac{11}{3} C_{2}\left(\epsilon \Delta_{0}\right)^{2 / 3} t^{2}, \quad t<t_{0}=\left(\frac{\Delta_{0}^{2}}{\epsilon}\right)^{1 / 3}
$$

where $C_{2}$ is the universal constant in the inertial range scaling law for the Eulerian second order velocity structure function with a well-known value of approximately $2.13(24)$. In the classical cascade model of turbulence, $t_{0}$ may be identified as the time for which the two fluid elements "remember" their initial relative velocity as they move in the same eddy of size $\Delta_{0}$. At times on the order of $t_{0}$, this eddy breaks up and the growth of the pair separation is expected to undergo a transition to Richardson-Obukhov scaling.

To distinguish between Batchelor and Richardson-Obukhov scaling, the inertial range must be large, so that there will be a large separation between the eddy turnover time $T_{L}$ and the Kolmogorov time $\tau_{\eta}$. To achieve such a wide range of scales, the turbulence level must be high, since $R_{\lambda} \sim\left(T_{L} / \tau_{\eta}\right)$. Based on evidence from direct numerical simulation (25), a turbulence level of at least $R_{\lambda}=600-700$ is required to see true inertial range scaling of a Lagrangian 
quantity like relative dispersion. Previous experimental and computational studies of dispersion have been limited by their low turbulence levels $\left(R_{\lambda}<300\right)(12-15,17)$ and have not been conclusive. High turbulence levels are obtained in kinematic simulation models (16), but such models may not be suited to the pair dispersion problem (26).

We show our measurements of relative dispersion for turbulence levels up to $R_{\lambda}=815$ in Fig. 2. We find that, for experimentally accessible initial separations, our data scales as $t^{2}$ for more than two decades in time, with no hint of Richardson-Obukhov $t^{3}$ scaling. This behavior holds throughout the entire inertial range, even for large initial separations (up to 70\% of the largest length scale of the turbulence). When we scale our relative dispersion data by the constant predicted by Batchelor, given in Eq. 1, the curves collapse onto a single $t^{2}$ power law. The line drawn in Fig. 2 is $(11 / 3) C_{2}\left(\epsilon \Delta_{0}\right)^{2 / 3} t^{2}$.

In Fig. 2, where time is plotted in units of $\tau_{\eta}$, the data for different initial separations deviate from the $t^{2}$ law at times that vary with $\Delta_{0}$. If, however, we scale time by Batchelor's $t_{0}=$ $\left(\Delta_{0}^{2} / \epsilon\right)^{1 / 3}$, as shown in Fig. 3, the data for each initial separation deviates from Batchelor's prediction at the same universal value of roughly $0.1 t_{0}$, irrespective of turbulence level.

For the quantities plotted in Figs. 2 and 3, we see no Richardson-Obukhov $t^{3}$ scaling. We have, however, also measured other statistics that, dimensionally, should obey the same scaling laws. One such quantity is exit time statistics (14). Our measurements of such statistics showed no clear $t^{3}$ behavior. Another measure of relative dispersion is shown in Fig. 4 in which we plot $\left(\left\langle\Delta^{2 / 3}\right\rangle-\Delta_{0}^{2 / 3}\right)$ scaled by $\Delta_{0}^{2 / 3}$ as a function of $t / t_{0}$. For small initial separations for which $\left(T_{L} / t_{0}\right)$ is of order 10 , we see a transition to a scaling law consistent with the RichardsonObukhov prediction for times greater than roughly $t_{0}$, irrespective of turbulence level. For larger initial separations for which $\left(T_{L} / t_{0}\right)$ is smaller, however, no such scaling is seen, as shown in the inset to Fig. 4. The existence of a transition at times of the order of $t_{0}$ shows once again that the initial separation is an important parameter for relative dispersion and cannot be neglected. 
In any practical application of relative dispersion, the initial source will have finite size and therefore a nonzero $\Delta_{0}$. Our data show that $t_{0}$ accurately quantifies the transition between the Batchelor scaling regime and the Richardson-Obukhov regime. Consequently, a clear $t^{3}$ scaling law requires not only a large separation between $T_{L}$ and $\tau_{\eta}$ but also a large separation between $T_{L}$ and $t_{0}$. For the initial separations accessible in our experiments, the maximum value of the ratio of $\left(T_{L} / t_{0}\right)$ was of order 10 , with no fully developed $t^{3}$ scaling. In order to apply the Richardson-Obukhov scaling law to a practical situation, then, $\left(T_{L} / t_{0}\right)$ must be much larger than 10, which implies the necessity of a high turbulence level.

For most flows on Earth, both natural and industrial, the turbulence levels are quite small; typically, $R_{\lambda}<1000$. Very turbulent atmospheric flows, such as warm clouds or the atmospheric boundary layer (27), have turbulence levels of about $R_{\lambda} \sim 10^{4}$. Even the most violent flows on Earth, such as plinian volcanic eruptions, have similar turbulence levels. If we consider a pair of particles with an initial separation of roughly $1 \mathrm{~m}$, such as might be found in the smokestack of an industrial plant, for a turbulence level of $R_{\lambda} \sim 10^{4},\left(T_{L} / t_{0}\right)$ is only about 30, assuming typical atmospheric flow parameters (28).

An important consequence of these results is that in almost all flows with industrial or biological significance the initial separation $\Delta_{0}$ will influence the subsequent spreading of the two fluid elements throughout the entire period of their turbulent superdiffusive separation. This can explain, for example, measurements of the decay of the fluctuations of a passive scalar injected into the flow (29). This decay becomes slower as the separation between two sources was increased. These results may in turn explain why the spatial arrangement of odor sources plays such an important role in the way crayfish and other crustaceans navigate their marine environments (5).

In summary, we observed that Batchelor's prediction is fulfilled for more than two decades in time at high turbulence levels. While our data may be somewhat contaminated by the in- 
homogeneity and anisotropy present in our specific flow, the observed scale collapse onto the Batchelor law appears very robust. In addition, we showed that the initial separation of the particle pair remains important in most flows in nature up to times of order $t_{0}$, which itself depends on the initial separation. We observed a transition near $t_{0}$ only when $\left(T_{L} / t_{0}\right)$ is of order 10 or larger. Therefore, a large separation between $T_{L}$ and $t_{0}$ is required to see a fully developed Richardson-Obukhov scaling regime, requiring a turbulence level beyond the reach of current experiments and higher than will occur in most practical situations.

The difference between Richardson-Obukhov and Batchelor scaling can have surprising consequences. Consider a leak of a highly toxic gas such as chlorine, widely used as a disinfectant, pesticide, and algaecide, and the first chemical weapon ever used in war. The United States Occupational Safety and Health Administration has specified that the acceptable level of gaseous chlorine exposure is 1 part per million. Recall that the local concentration decay rate is related to relative dispersion (8). Using the Richardson-Obukhov law, the variance of the chlorine concentration decays as $t^{-9 / 2}$ as the gas disperses into the surrounding air. Batchelor scaling, however, predicts that the concentration variance will decay as $t^{-3}$. A simple calculation then shows that, in the initial stages, the local concentration could decay to safe levels 20 times slower when Batchelor scaling is assumed. Our results therefore are of immediate application for the health and safety of those who work with toxic chemicals.

\section{References and Notes}

1. B. I. Shraiman, E. D. Siggia, Nature 405, 639 (2000).

2. M. Huber, J. C. McWilliams, M. Ghil, J. Atmos. Sci. 58, 2377 (2001).

3. P. S. Berloff, J. C. McWilliams, A. Bracco, J. Phys. Oceanogr. 32, 764 (2002).

4. J. B. Joergensen, J. Mann, S. Ott, H. L. Pécseli, J. Trulsen, Phys. Fluids 17, 035111 (2005). 
5. M. C. Wolf, R. Voigt, P. A. Moore, J. Chem. Ecol. 30, 501 (2004).

6. P. Moore, J. Crimaldi, J. Marine Syst. 49, 55 (2004).

7. S. Edouard, B. Legras, F. Lefèvre, R. Eymard, Nature 384, 444 (1996).

8. M. Nelkin, R. M. Kerr, Phys. Fluids 24, 1754 (1981).

9. B. Sawford, Annu. Rev. Fluid Mech. 33, 289 (2001).

10. L. F. Richardson, Proc. R. Soc. Lond. A 110, 709 (1926).

11. G. K. Batchelor, Q. J. R. Meterol. Soc. 76, 133 (1950).

12. P. K. Yeung, Phys. Fluids 6, 3416 (1994).

13. S. Ott, J. Mann, J. Fluid Mech. 422, 207 (2000).

14. G. Boffetta, I. M. Sokolov, Phys. Rev. Lett. 88, 094501 (2002).

15. P. K. Yeung, M. S. Borgas, J. Fluid Mech. 503, 93 (2004).

16. F. Nicolleau, G. Yu, Phys. Fluids 16, 2309 (2004).

17. L. Biferale, et al., Phys. Fluids 17, 115101 (2005).

18. A. La Porta, G. A. Voth, A. M. Crawford, J. Alexander, E. Bodenschatz, Nature 409, 1017 (2001).

19. G. A. Voth, A. La Porta, A. M. Crawford, J. Alexander, E. Bodenschatz, J. Fluid Mech. 469, 121 (2002).

20. M. Virant, T. Dracos, Meas. Sci. Technol. 8, 1539 (1997). 
21. N. T. Ouellette, H. Xu, E. Bodenschatz, Exp. Fluids (2005). DOI: 10.1007/s00348-0050068-7.

22. A. N. Kolmogorov, Dokl. Akad. Nauk SSSR 30, 301 (1941).

23. A. M. Obukhov, Izv. Akad. Nauk SSSR 5, 453 (1941).

24. K. R. Sreenivasan, Phys. Fluids 7, 2778 (1995).

25. P. K. Yeung, Annu. Rev. Fluid Mech. 34, 115 (2002).

26. D. J. Thomson, B. J. Devenish, J. Fluid Mech. 526, 277 (2005).

27. S. Kurien, K. R. Sreenivasan, Phys. Rev. E 64, 056302 (2001).

28. R. A. Shaw, Annu. Rev. Fluid Mech. 35, 183 (2003).

29. Z. Warhaft, J. L. Lumley, J. Fluid Mech. 88, 659 (1978).

30. This research is supported by the Physics Division of the National Science Foundation and by the Max Planck Society. We thank L. Collins, G. Falkovich, J. Hunt, J. Schumacher, D. Vincenzi, and Z. Warhaft for helpful discussions and suggestions over the course of this work. 

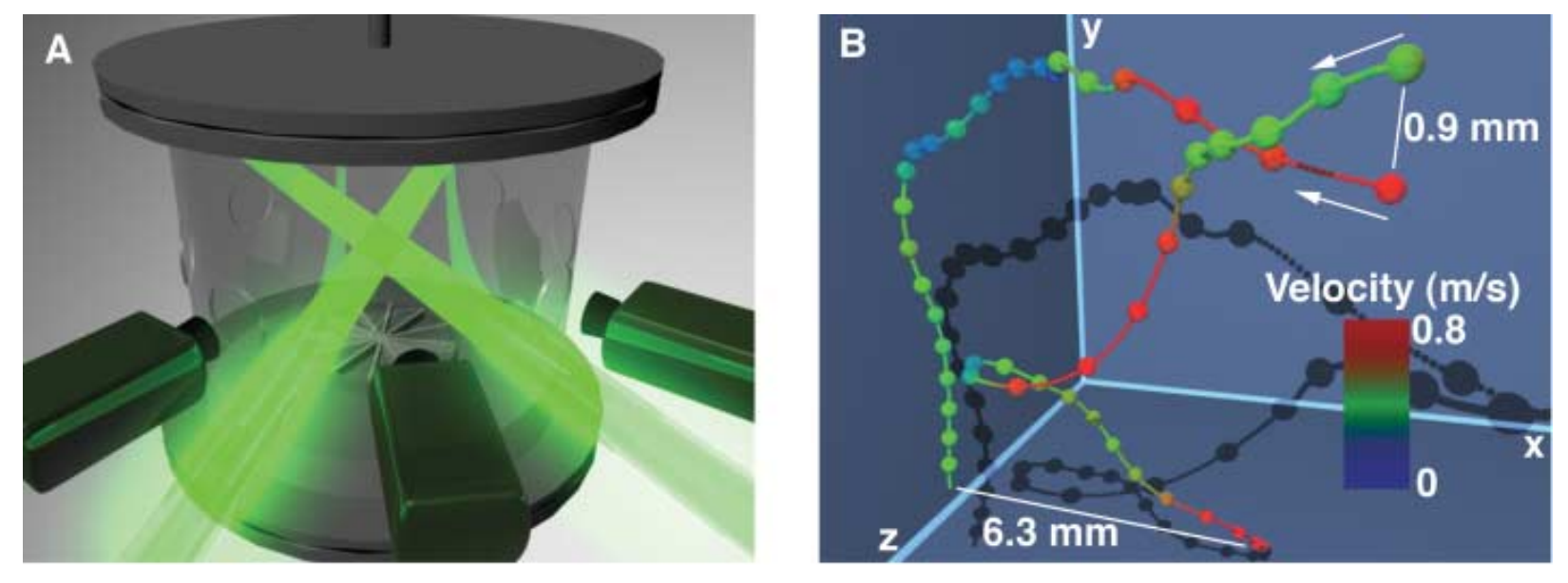

Figure 1: (a) Sketch of the experimental setup. Three high-speed cameras are used to record the 3D tracks of tracer particles in intense turbulence. The particles are illuminated by two high- power lasers. (b) A pair of measured particle trajectories at $R \lambda=690$. The small spheres mark every other measured position of the particle's, and are separated by $0.074 \mathrm{~ms}\left(\approx \mathrm{T}_{\eta} / 13\right)$ in time; the large spheres mark every 30 th position. The color of the spheres indicates the magnitude of each particles absolute velocity in units of $\mathrm{m} / \mathrm{s}$. The particles enter the measurement volume as indicated by the arrows, and separate under the influence of the turbulence. 


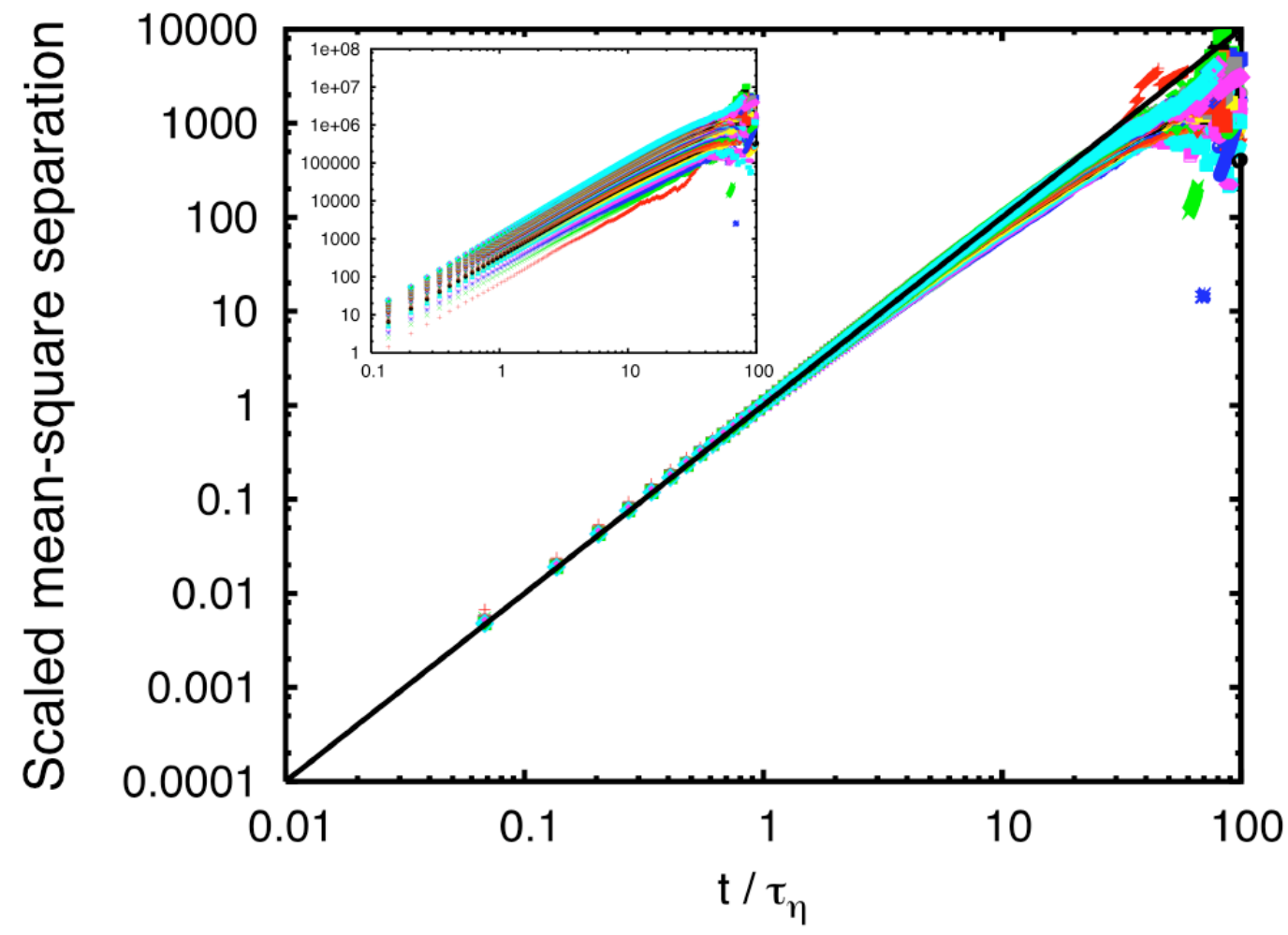

Figure 2: Evolution of the mean-square particle separation. The mean-square separation be- tween particle pairs is plotted against time for fifty different initial separations at a turbulence level of $R \lambda=815$, with the time axis normalized by the Kolmogorov scales. Each curve repre- sents a bin of initial separations $1 \mathrm{~mm}$ wide $(\approx 43 \eta)$, ranging from $0-1 \mathrm{~mm}$ to 49-50 $\mathrm{mm}$. The curves are scaled by the constant $(11 / 3) \mathrm{C}_{2}(\varepsilon \Delta 0) 2 / 3$ (Eq. 1). The data collapse onto a single universal power law. The bold line is the power law predicted by Batchelor (11). We note that since the smallest $\Delta$ o measured is not in the inertial range, we do not expect it to scale perfectly as t2, and indeed it does not scale as well as the larger $\Delta$. The inset shows the same curves scaled simply by the Kolmogorov length, for which we see no scale collapse. For both plots, we see no Richardson-Obukhov t3 scaling. 


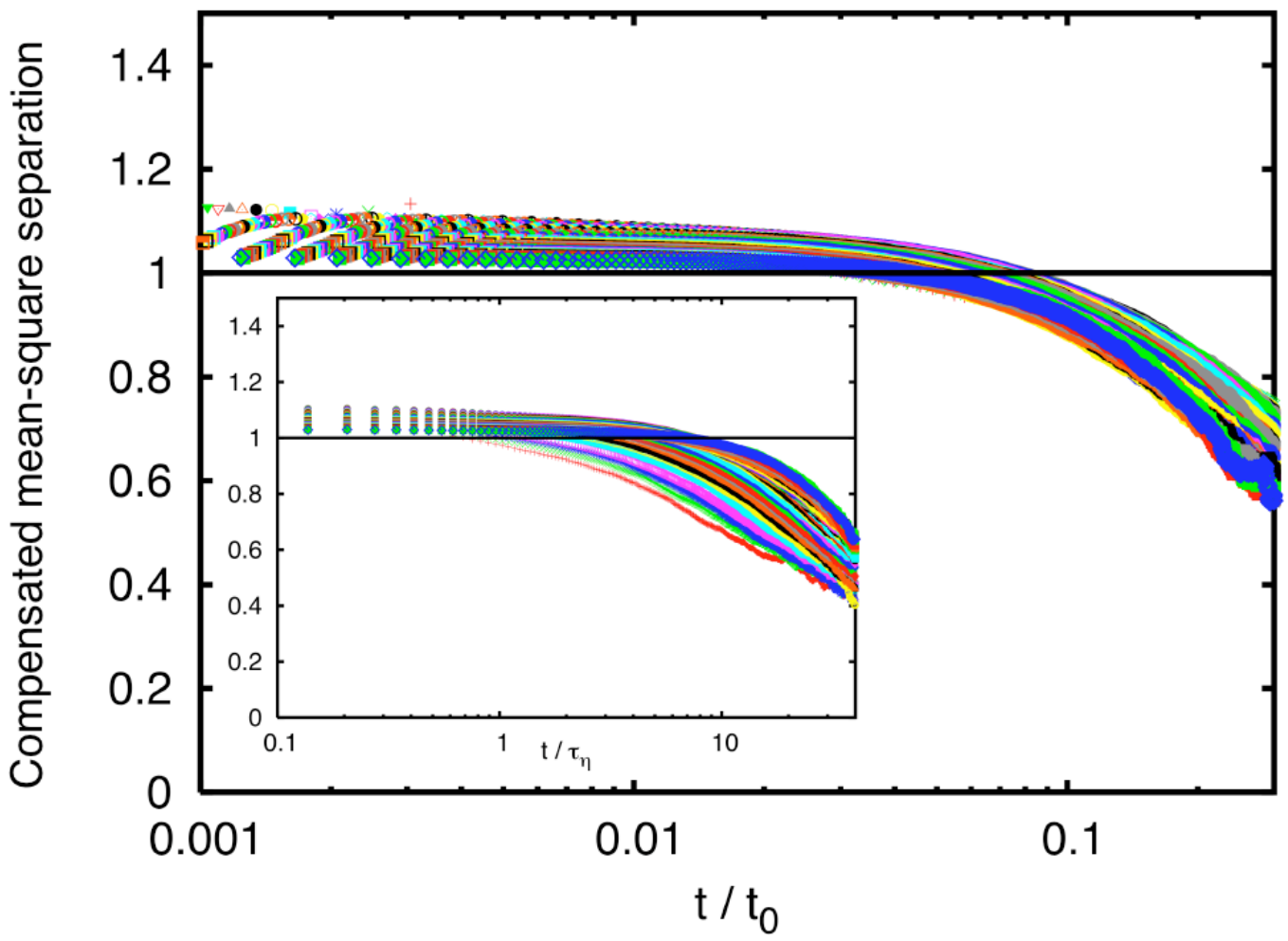

Figure 3: Mean-square separation with time scaled by to. The mean-square separation at $\mathrm{R}_{\lambda}=815$ compensated by Batchelor's scaling law (Eq. 1) is plotted against time in units of $t_{0}=\left(\Delta_{20} / \varepsilon\right)_{1 / 3}$. Plotted in this way, a plateau corresponds to Batchelor scaling. The inset shows the same compensated data plotted against time scaled by the Kolmogorov time. The data clearly collapses significantly better with time scaled by to. The data begins to deviate from a t2 power law at a universal time of about 0.1 to. 


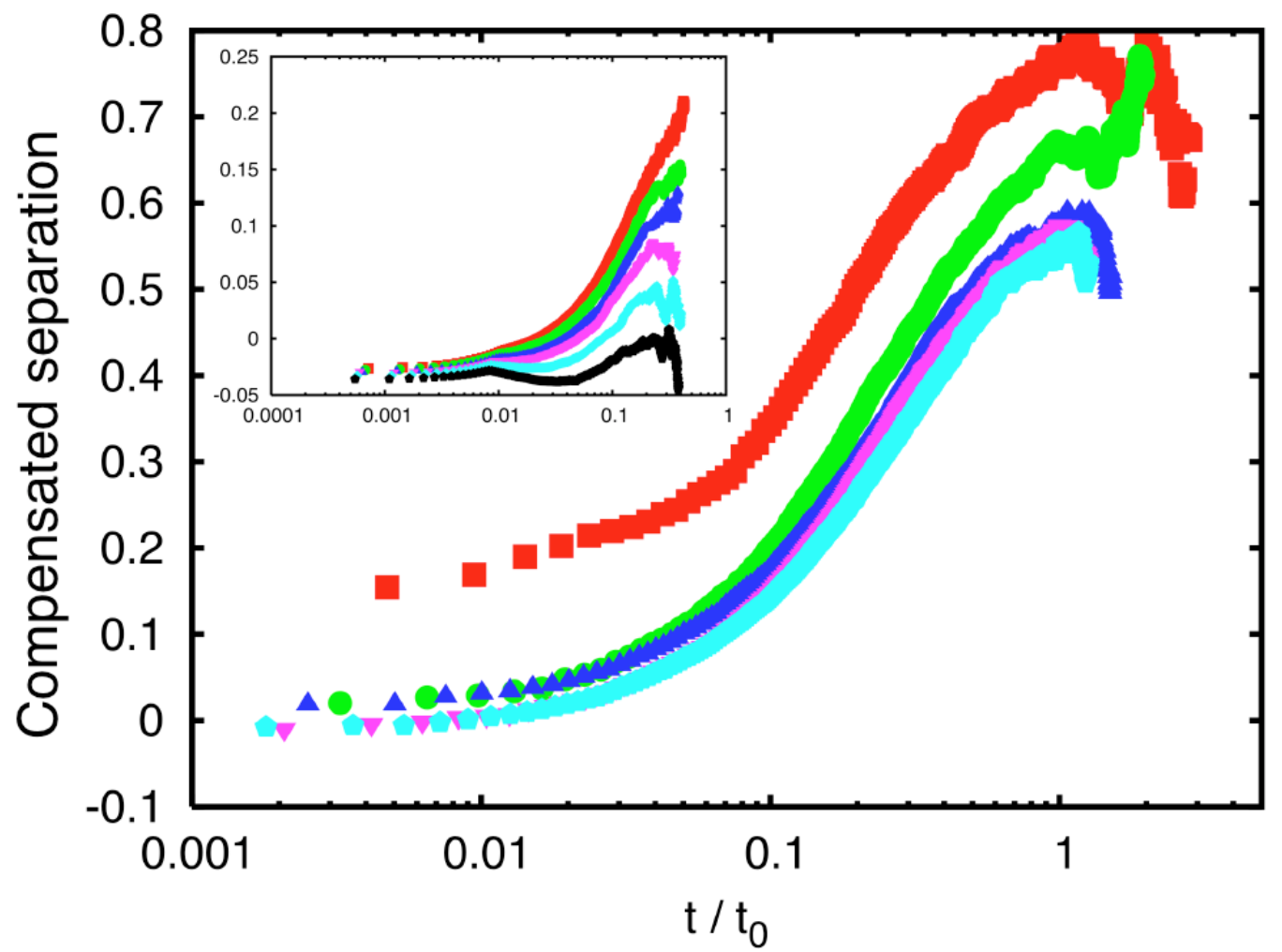

Figure 4: $\left(\left\langle\Delta(\mathrm{t})_{2 / 3}\right\rangle-\Delta_{2 / 3}\right)$ scaled by $\Delta_{2 / 3}$ and compensated by $\mathrm{t} / \mathrm{t}$. The data are plotted 000 against t/to; a plateau denotes Richardson-Obukhov-like scaling. The initial separation in10creases from $1 \mathrm{~mm}(\approx 43 \eta)$ for the top curve to $5 \mathrm{~mm}(\approx 215 \eta)$ for the bottom curve, and $(\mathrm{T}\llcorner/ \mathrm{t})$ is of order 10 . The inset shows the same quantity plotted against $t / t$ for larger initial separations, ranging from $20 \mathrm{~mm}(\approx 860 \eta)$ for the top curve to $30 \mathrm{~mm}(\approx 1290 \eta)$ for the bottom curve. 